\title{
Dirac cone and pseudogapped density of states in the topological half-Heusler compound YPtBi
}

\author{
A. Kronenberg, ${ }^{1}$ J. Braun, ${ }^{2}$ J. Minár, ${ }^{2,3}$ H.-J. Elmers, ${ }^{1}$ D. Kutnyakhov, ${ }^{1}$ A. V. Zaporozhchenko, ${ }^{1}$ R. Wallauer, ${ }^{1}$ S. Chernov, ${ }^{1}$ \\ K. Medjanik, ${ }^{1}$ G. Schönhense, ${ }^{1}$ M. Kläui, ${ }^{1}$ S. Chadov, ${ }^{4}$ H. Ebert, ${ }^{2}$ and M. Jourdan ${ }^{1, *}$ \\ ${ }^{1}$ Institute of Physics, Johannes Gutenberg-University, 55099 Mainz, Germany \\ ${ }^{2}$ Department Chemie, Ludwig-Maximilians-Universität München, Butenandtstrasse 11, 81377 München, Germany \\ ${ }^{3}$ New Technologies-Research Center, University of West Bohemia, Univerzitni 8, 30614 Pilsen, Czech Republic \\ ${ }^{4}$ Max-Planck-Institut für Chemische Physik fester Stoffe, 01187 Dresden, Germany \\ (Received 4 July 2016; revised manuscript received 14 September 2016; published 6 October 2016)
}

\begin{abstract}
Topological insulators (TIs) are exciting materials, which exhibit unprecedented properties, such as helical spin-momentum locking, which leads to large torques for magnetic switching and highly efficient spin current detection. Here we explore the compound YPtBi, an example from the class of half-Heusler materials, for which the typical band inversion of topological insulators was predicted. We prepared this material as thin films by conventional cosputtering from elementary targets. By in situ time-of-flight momentum microscopy, a Dirac conelike surface state with a Dirac point $\simeq 300 \mathrm{meV}$ below the Fermi energy was observed, in agreement with electronic structure-photoemission calculations. Only little additional spectral weight due to other states was observed at $E_{F}$, which corroborates the identification of the topologically protected surface state and is highly relevant for spintronics applications.
\end{abstract}

DOI: 10.1103/PhysRevB.94.161108

Three-dimensional topological insulators are insulating in the bulk, but strong spin-orbit coupling enables a special electronic structure with inverted bands that results in the formation of a topologically protected single Dirac conelike surface state [1-3]. As in such materials the orientation of the spins is fixed relative to the direction of a charge current carried by the surface state, its potential for spintronics applications is evident. A perfect conversion between a spin and a charge current thus would ultimately enable high spin currents and corresponding spin torques generated from a charge current flowing in the topological state. Indeed, exceptionally strong spin-transfer torques generated by the topological insulator $\mathrm{Bi}_{2} \mathrm{Se}_{3}$ and related compounds were recently reported [4-7]. Thus, considering future applications such as spin-orbit torque magnetic random access memory (SOT-MRAM) [8], topological insulators should well exceed the switching efficiency reached by conventional materials such as Pt and Ta [9-11]. As pointed out by Mellnik et al. [4], this potential application utilizes the helical spin-momentum locking of the topological insulator surface state and does not depend on having zero bulk conductivity within the topological insulator. Furthermore, the inverse effect, namely, the conversion of a spin current into a charge current, will allow for the ultimately sensitive detection of spin currents generated by spin pumping or the spin Seebeck effect [12].

However, for both applications, the toxicity of Se makes the incorporation of $\mathrm{Bi}_{2} \mathrm{Se}_{3}$ and related materials in real devices challenging, and the deposition techniques and conditions tend to be sophisticated and incompatible with industrial environments. Thus, new topological insulators consisting of generally nontoxic components suitable for industrial production processes, including high-temperature annealing steps, are required.

*Jourdan@uni-mainz.de
A class of materials where topological insulator compounds have been predicted are half Heuslers [13]. These represent ideal candidate materials for such applications. Beyond the SOT-MRAM and spin current detection and conversion-related motivation described above, they include superconducting [14,15], antiferromagnetic [16], half-metallic ferromagnetic $[17,18]$, and semiconducting [19] compounds, which makes them most interesting for various applications and basic science related fields of research.

Within the class of half-Heusler compounds, a large number of materials was predicted to show the necessary inversion of the $\Gamma_{6}$ and $\Gamma_{8}$ bands [20-23]. The electronic structure of the semiconducting 18-valence-electron Heusler compounds is strongly related to that of CdTe and $\mathrm{HgTe}$ [20]. As the ternary $X Y Z$ Heusler class comprises many semiconductors and semimetals, it provides large flexibility in design by tailoring the band gap and the spin-orbit coupling. One of the most attractive aspects of half-Heusler compounds is the tunability of the insulating gap over a wide energy range, from about $4 \mathrm{eV}$ (e.g., LiMgN) down to zero (e.g., ScPtBi), by choosing $X Y Z$ combinations with different electronegativities of the constituents and with different lattice constants [22]. However, the calculated band structures of the undistorted half-Heusler compounds with band inversion show bulk states at the Fermi energy $E_{F}[20,21]$, i.e., they are topological semimetals, not insulators.

Some experimental indications of the topological properties of half-Heusler compounds were published in the last years [24-30], but only very recently first direct experimental evidence was reported: Logan et al. [31] provided evidence for a Dirac cone well above $E_{F}$ of $\operatorname{LuPtSb}(001)$ by the extrapolation of linearly dispersing bands below $E_{F}$ observed by angular-resolved photoemission spectroscopy (ARPES). Liu et al. [32] reported the observation of a Dirac conelike feature well below $E_{F}$ by investigating bulk single crystals of LuPtBi by ARPES. Additionally, pronounced additional spectral weight due to other states was observed at $E_{F}$. However, such additional states will strongly influence the 


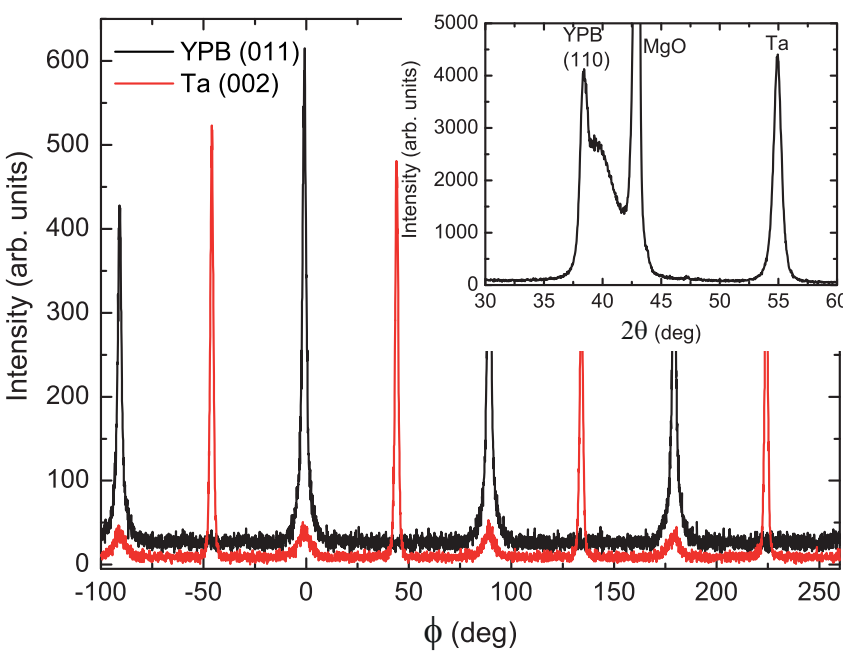

FIG. 1. XRD $\phi$ scans of in-plane peaks of a YPtBi(110) thin film and of in-plane peaks of the Ta(100) buffer layer. The Ta peaks show the expected fourfold symmetry of an epitaxial layer. The YPtBi $(01 \overline{1})$ and equivalent peaks, which would be observed twofold for a fully epitaxial layer, appear to be fourfold, indicating two orientations of in-plane ordered domains. The inset shows an XRD $\Theta / 2 \Theta$ scan with the specular $\mathrm{YPtBi}(110)$ and $\mathrm{Ta}(200)$ peaks of the thin film grown on a $\mathrm{MgO}(100)$ substrate.

effective transport spin polarization relevant for spintronics and are in general expected to interfere with the overlapping Dirac cone.

Here we show the results of in situ time-of-flight (TOF) momentum microscopy of the half-Heusler compound YPtBi, and directly demonstrate the presence of a Dirac cone representing a topologically protected surface state, together with only little additional spectral weight at $E_{F}$ due to other states. We compare the experimental results with one-step photoemission calculations, showing good agreement.

We investigated the surface band structure of the YPtBi thin films $(<10 \mathrm{~nm})$ in situ by TOF-momentum microscopy. Based on our experience with photoemission experiments on other Heusler compounds [18,33], a setup was realized which allows the direct ultrahigh vacuum transfer of the thin film samples from the deposition to the spectroscopy chamber. The TOF-momentum microscope [34-36] measures a threedimensional (3D) data array of the energy and momentum dependent photoemission intensity $I\left(E, k_{x}, k_{y}\right)$. The instrument utilizes an electron optical reciprocal image representing the distribution of the transversal momentum components $k_{\|}$, which are conserved in the photoemission process. The energy resolution is obtained by a time-of-flight measurement of the photoelectrons excited by a pulsed, frequency-quadrupled Ti:sapphire laser resulting in a photon energy of $h v=6.2 \mathrm{eV}$. Due to this, low photon energy surface states dominate the photoemission spectra in this particular case. The photon angle of incidence ( $p$-polarized light) amounted to $68^{\circ}$ from the sample normal, i.e., the (110) direction of YPtBi. An aperture in the microscope optics allows the selection of a circular sample region of a diameter of $7 \mu \mathrm{m}$ from which the photoemission intensity $I\left(E, k_{x}, k_{y}\right)$ is collected.

Electronic structure calculations were performed by use of a generalized-gradient approximation (GGA) of the density functional theory (DFT) [37]. For the exchange and correlation potentials we applied the Perdew, Burke, and Enzerhof parametrization [38]. The corresponding first-principles calculations were done in a fully relativistic mode by solving the corresponding Dirac equation. This was achieved by using the spin-polarized relativistic multiple-scattering or KorringaKohn-Rostoker formalism (SPRKKR) as implemented in the Munich SPRKKR program package [39].

Our spectroscopic analysis is based on the fully relativistic one-step model of photoemission [40] in its spin-density matrix formulation. This approach allows for the proper description of the $k$ dependence of the photoemission intensity and spin polarization vector [41]. The initial and final states are constructed in the framework of spin-polarized low-energy electron diffraction (SPLEED) theory, where the final state is represented by a so-called time-reversed SPLEED state [40,42]. For the explicit photoemission calculations, we added to the semi-infinite stack of atomic layers a surface barrier by use of a Rundgren-Malmström-type surface potential [43], which is included in the SPLEED formalism as an additional (a)

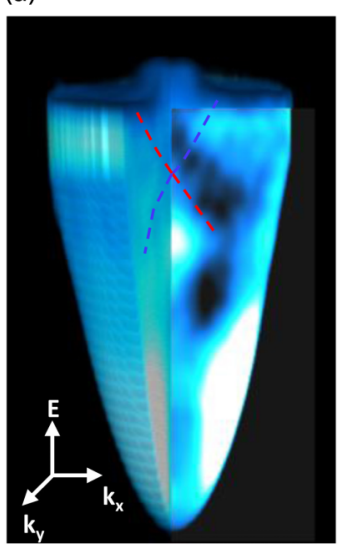

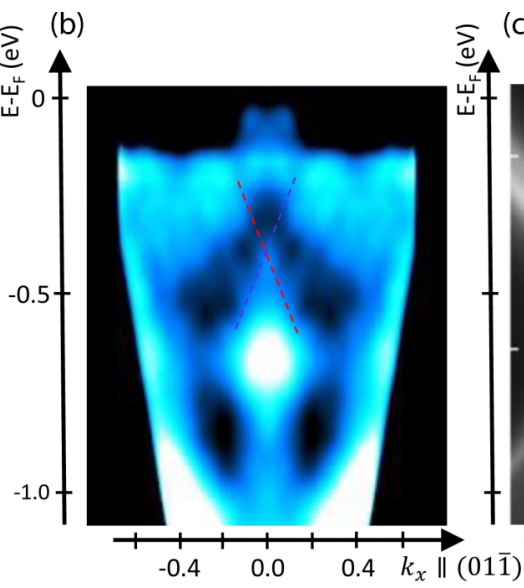
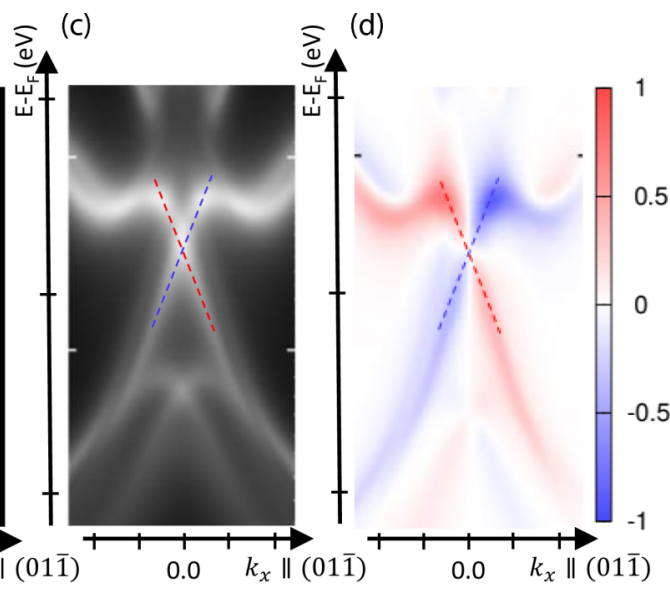

FIG. 2. (a) Full representation and (b) constant $k_{y}$ sections through the experimental 3D data array of energy and momentum dependent photoemission intensity $I\left(E, k_{x}, k_{y}\right)$. (c) shows the corresponding photoelectron intensity and (d) photoelectron spin polarization [color coded projection along $\left.k_{y} \|(001)\right]$. The dashed lines serve to guide the eye, indicating the Dirac cone. 
layer. This allows for the correct description of the asymptote of a potential away from the crystal surface. As a consequence, an additional surface contribution of the total photocurrent results, which accounts explicitly for the energetics and dispersion of all surface-related features. Furthermore, the relative intensities of surface-related spectral distributions are quantitatively accounted for by calculating the corresponding matrix elements in the surface region. This procedure, in which energy and momentum conservation are naturally included [40,44], is described in detail, e.g., in Ref. [45]. Considering impurity scattering, a small constant imaginary value of $V_{i 1}=0.03 \mathrm{eV}$ was used for the initial state, which describes the finite lifetime. Lifetime effects in the final state are accounted for by the imaginary part of the inner potential. A constant imaginary value of $V_{i 2}=1.2 \mathrm{eV}$ has been chosen in a phenomenological way to account for the inelastic mean free path of the photoelectrons resulting from the experimental excitation energy of $6.2 \mathrm{eV}$. The angle of incidence and light polarization were adopted to the experimental geometry.

By cosputtering from three elementary targets, we prepared semiepitaxial YPtBi thin films. The three components were deposited with the rates of $r(\mathrm{Y})=0.45 \mathrm{~nm} / \mathrm{s}, r(\mathrm{Pt})=$ $0.34 \mathrm{~nm} / \mathrm{s}$, and $r(\mathrm{Bi})=0.75 \mathrm{~nm} / \mathrm{s}$ on a $\mathrm{MgO}(100)$ substrate with a $\mathrm{Ta}(100)$ buffer layer (thickness $20 \mathrm{~nm}$ ) held at $T=$ $615^{\circ} \mathrm{C}$.

X-ray diffraction (XRD) $\Theta / 2 \Theta$ scans (inset of Fig. 1) show the (110) peak of YPtBi(10 nm) and the (200) peak of the $\mathrm{Ta}(20 \mathrm{~nm})$ buffer layer, which are oriented perpendicular to the substrate surface.

A grazing incidence XRD $\phi$ scan (azimuth angle) with the Bragg angle $\Theta$ selecting the $(01 \overline{1})$ and equivalent in-plane peaks revealed a fourfold in-plane symmetry (Fig. 1). As the (110) plane of YPtBi has a twofold symmetry, this means that the thin films, averaging $\mathrm{a} \simeq 1 \mathrm{~mm}$ sized area, consist of grains with two different in-plane orientations rotated by $90^{\circ}$ with respect to each other. Within the experimental error of our XRD experiments, we do not observe any strain in our samples.

Scanning electron microscopy (not shown) demonstrated that the thin film surface is free of segregations. Figure 2(a) shows a representation of the 3D data array of energy and momentum dependent photoemission intensity $I\left(E, k_{x}, k_{y}\right)$. It is obvious that at $E_{F}$ only little spectral weight around the $\Gamma$ point is observed, resembling the pseudogapped band structure of a semimetal. Figure 2(b) depicts a cut through this data with $k_{y}=0$, and Figs. 2(c) and 2(d) show the corresponding calculated photoelectron intensity and spin polarization. The helical spin-locked Dirac state displays as two linearly dispersing states in the $E(k)$ cut with a crossing point (Dirac point) at $E-E_{F}=-0.3 \mathrm{eV}$ (indicated by the dotted blue and red lines). This feature shows up both in the experimental result as well as in the calculation (Fig. 2). The high intensity observed at $E-E_{F}=-0.6 \mathrm{eV}(-0.7 \mathrm{eV}$ in the calculation) originates from a spin split Rashba surface resonance. The additional electronic states parallel to the Dirac state that do not show up in the calculation may result from the finite thickness of the sample, leading to quantum size effects along $k_{z}$.

According to the calculation, the spin polarization [Fig. 2(d)] points perpendicularly to the in-plane momentum component. While the spin polarization of the ground-state spectral function amounts to $100 \%$ near the Dirac point, the photoemission calculation results in reduced values for $p$-polarized light.

The position on the sample of the $7 \mu \mathrm{m}$ spot from which the TOF-momentum microscope collects the 3D data array of energy and momentum dependent photoemission intensity $I\left(E, k_{x}, k_{y}\right)$ was selected to optimize the contrast of constantenergy sections. This resulted in $I\left(E, k_{x}, k_{y}\right)$ constant-energy sections with twofold symmetry, i.e., from the two possible in-plane orientations of the YPtBi(110) crystallites identified by XRD, only one was selected.

Figure 3 (left column) shows constant-energy sections through $I\left(E, k_{x}, k_{y}\right)$, symmetrized according to the crystal symmetry. For comparison, the central column shows the corresponding calculated spin-integrated photoemission intensity.

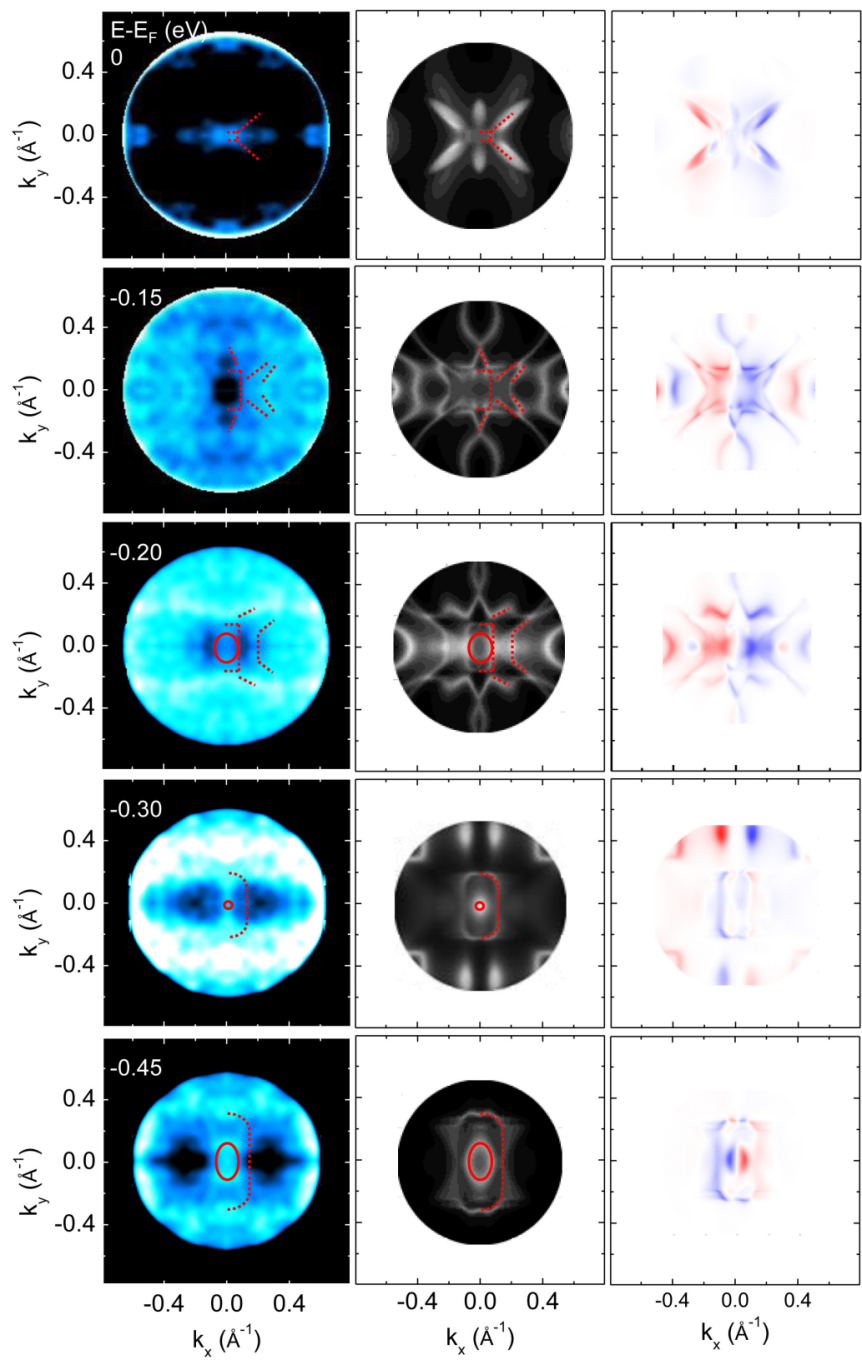

FIG. 3. Left column: Constant-energy sections through the experimental 3D data array of energy and momentum dependent photoemission intensity $I\left(E, k_{x}, k_{y}\right)$. For image improvement the experimental data was symmetrized according to the crystal symmetry. Central column: Corresponding calculated intensity. Right column: Corresponding calculated spinpolarization (color coded projection along $k_{y}$ ), color bar see Fig. 2 . 
The right column shows the calculated spin polarization. The dashed lines in the constant-energy sections serve to guide the eye, indicating common features of the experimental and theoretical data.

At the Fermi energy, photoelectron intensity is observed mainly around the gamma point, which agrees well with the corresponding photoemission calculation. The qualitative agreement between the measured constant-energy section at the Fermi energy $I\left(E_{F}, k_{x}, k_{y}\right)$ and the corresponding calculations indicating a high spin polarization is obvious, although the relative intensities of the features differ. Such limitations concerning the $k$-dependent intensity of photoemission are common and observed for materials such as $\operatorname{Mo(110)}$ as well [36].

Below the Fermi energy, the experimentally determined constant-energy sections show more structures than the corresponding calculations. However, the main characteristic features of the theoretical sections, as indicated by the dashed red lines in Fig. 3, can be found within the experimental data. At $300 \mathrm{meV}$ below the Fermi energy, a spotlike photoemission intensity feature is found at the $\Gamma$ point, which develops into an oval band around the $\Gamma$ point for increased as well as for decreased binding energies, as indicated by the solid red lines in Fig. 3. This feature corresponds to the Dirac cone discussed above. Additionally, the dispersion of other states is observed, which were identified as surface states by the application of the determinant criterion $[46,47]$. Our calculation predicts a high spin polarization of these states as well.

In conclusion, by TOF-momentum microscopy investigations of cosputtered $\mathrm{YPtBi}(110)$ thin films and comparison with corresponding calculations, a helical spin-locked Dirac state was identified, providing direct experimental evidence for the realization of a topologically protected surface state in these half-Heusler thin films. At $E_{F}$ only a little spectral weight around the $\Gamma$ point was observed, corresponding to a pseudogapped surface band structure. This corroborates the identification of the spin-polarized linearly dispersing branches as topologically protected surface states. As the Dirac cone dominates the surface density of states of YPtBi(110) at $E_{F}$, further effort may result in novel spintronic devices with industry and environmentally compatible components beyond what is achievable with the currently available materials.

This work was partially supported by the DFG via the priority program Topological Insulators (E1172/18-1) and via project Jo404/9-1.
[1] L. Fu, C. L. Kane, and E. J. Mele, Phys. Rev. Lett. 98, 106803 (2007).

[2] J. E. Moore and L. Balents, Phys. Rev. B 75, 121306(R) (2007).

[3] R. Roy, Phys. Rev. B 79, 195322 (2009).

[4] A. R. Mellnik, J. S. Lee, A. Richardella, J. L. Grab, P. J. Mintun, M. H. Fischer, A. Vaezi, A. Manchon, E.-A. Kim, N. Samarth, and D. C. Ralph, Nature (London) 511, 449 (2014).

[5] Y. Shiomi, K. Nomura, Y. Kajiwara, K. Eto, M. Novak, K. Segawa, Y. Ando, and E. Saitoh, Phys. Rev. Lett. 113, 196601 (2014).

[6] Y. Fan, P. Upadhyaya, X. Kou, M. Lang, S. Takei, Z. Wang, J. Tang, L. He, L.-T. Chang, M. Montazeri, G. Yu, W. Jiang, T. Nie, R. N. Schwartz, Y. Tserkovnyak, and K. L. Wang, Nat. Mater. 13, 699 (2014).

[7] A. A. Baker, A. I. Figueroa, L. J. Collins-McIntyre, G. van der Laan, and T. Hesjedal, Sci. Rep. 5, 7907 (2015).

[8] A. Brataas and K. M. D. Hals, Nat. Nanotechnol. 9, 86 (2014).

[9] L. Liu, O. J. Lee, T. J. Gudmundsen, D. C. Ralph, and R. A. Buhrman, Phys. Rev. Lett. 109, 096602 (2012).

[10] G. Yu, P. Upadhyaya, Y. Fan, J. G. Alzate, W. Jiang, K. L. Wong, S. Takei, S. A. Bender, L.-T. Chang, Y. Jiang, M. Lang, J. Tang, Y. Wang, Y. Tserkovnyak, P. K. Amiri, and K. L. Wang, Nat. Nanotechnol. 9, 548 (2014).

[11] R. Lo Conte, A. Hrabec, A. P. Mihai, T. Schulz, S.-J. Noh, C. H. Marrows, T. A. Moore, and M. Kläui, Appl. Phys. Lett. 105, 122404 (2014).

[12] Z. Jiang, Ph.D. thesis, University of California, 2015, https://oatd.org/oatd/record?record=california\%3Aqt8st569mj

[13] F. Casper, T. Graf, S. Chadov, B. Balke, and C. Felser, Semicond. Sci. Technol. 27, 063001 (2012).

[14] G. Goll, M. Marz, A. Hamann, T. Tomanic, K. Grube, T. Yoshino, and T. Takabatake, Physica B 403, 1065 (2008).
[15] Y. Nakajima, R. Hu, K. Kirshenbaum, A. Hughes, P. Syers, X. Wang, K. Wang, R. Wang, S. R. Saha, D. Pratt, J. W. Lynn, and J. Paglione, Sci. Adv. 1, e1500242 (2015).

[16] T. Jeong, R. Weht, and W. E. Pickett, Phys. Rev. B 71, 184103 (2005).

[17] R. A. de Groot, F. M. Mueller, P. G. van Engen, and K. H. J. Buschow, Phys. Rev. Lett. 50, 2024 (1983).

[18] M. Jourdan, J. Minár, J. Braun, A. Kronenberg, S. Chadov, B. Balke, A. Gloskovskii, M. Kolbe, H. J. Elmers, G. Schönhense, H. Ebert, C. Felser, and M. Kläui, Nat. Commun. 5, 3974 (2014).

[19] S. Ögüt and K. M. Rabe, Phys. Rev. B 51, 10443 (1995).

[20] S. Chadov, X. Qi, J. Kübler, G. H. Fecher, C. Felser, and S. C. Zhang, Nat. Mater. 9, 541 (2010).

[21] H. Lin, L. A. Wray, Y. Xia, S. Xu, S. Jia, R. J. Cava, A. Bansil, and M. Z. Hasan, Nat. Mater. 9, 546 (2010).

[22] B. Yan and A. de Visser, MRS Bull. 39, 859 (2014).

[23] S.-Y. Lin, M. Chen, X.-B. Yang, Y.-J. Zhao, S.-C. Wu, C. Felser, and B. Yan, Phys. Rev. B 91, 094107 (2015).

[24] N. P. Butch, P. Syers, K. Kirshenbaum, A. P. Hope, and J. Paglione, Phys. Rev. B 84, 220504 (2011).

[25] Q. Pavlosiuk, D. Kaczorowski, and P. Wisniewski, Sci. Rep. 5, 9158 (2015).

[26] R. Shan, S. Ouardi, G. H. Fecher, L. Gao, A. Kellock, A. Gloskovskii, C. E. ViolBarbosa, E. Ikenaga, C. Felser, and S. S. P. Parkin, Appl. Phys. Lett. 101, 212102 (2012).

[27] T. Miyawaki, N. Sugimoto, N. Fukatani, T. Yoshihara, K. Ueda, N. Tanaka, and H. Asano, J. Appl. Phys. 111, 07E327 (2012).

[28] W. Wang, Y. Du, E. Liu, Z. Liu, and G. H. Wu, J. Appl. Phys. 112, 103910 (2012).

[29] R. Shan, S. Ouardi, G. H. Fecher, L. Gao, A. Kellock, K. P. Roche, M. G. Samant, C. E. ViolBarbosa, E. Ikenaga, C. Felser, and S. S. P. Parkin, Appl. Phys. Lett. 102, 172401 (2013). 
[30] R. Shan, E. V. Vilanova, J. Qin, F. Casper, G. H. Fecher, G. Jakob, and C. Felser, Phys. Status Solidi 7, 145 (2012).

[31] J. A. Logan, S. J. Patel, S. D. Harrington, C. M. Polley, B. D. Schultz, T. Balasubramanian, A. Janotti, A. Mikkelsen, and C. J. Palmstrøm, Nat. Commun. 7, 11993 (2016).

[32] Z. Liu, L. Yang, S.-C. Wu, C. Shekhar, J. Jiang, H. Yang, Y. Zhang, S.-K. Mo, Z. Hussain, B. Yan, C. Felser, and Y. Chen, arXiv:1602.05633 [cond-mat.mtrl-sci] (2016).

[33] M. Kolbe, S. Chadov, E. A. Jorge, G. Schönhense, C. Felser, H. J. Elmers, M. Kläui, and M. Jourdan, Phys. Rev. B 86, 024422 (2012).

[34] A. Winkelmann, A. A. Ünal, C. Tusche, M. Ellguth, C.-T. Chiang, and J. Kirschner, New J. Phys. 14, 083027 (2012).

[35] G. Schönhense, K. Medjanik, and H.-J. Elmers, J. Electron Spectrosc. Relat. Phenom. 200, 94 (2015).

[36] S. V. Chernov, K. Medjanik, C. Tusche, D. Kutnyakhov, S. A. Nepijko, A. Oelsner, J. Braun, J. Minár, S. Borek, H. Ebert, H. J. Elmers, J. Kirschner, and G. Schönhense, Ultramicroscopy 159, 453 (2015)
[37] P. Hohenberg and W. Kohn, Phys. Rev. 136, B864 (1964); W. Kohn and L. J. Sham, ibid. 140, A1133 (1965); L. J. Sham and W. Kohn, ibid. 145, 561 (1966).

[38] J. P. Perdew, K. Burke, and M. Ernzerhof, Phys. Rev. Lett. 77, 3865 (1996).

[39] H. Ebert, D. Ködderitzsch, and J. Minár, Rep. Prog. Phys. 74, 096501 (2012).

[40] J. Braun, Rep. Prog. Phys. 59, 1267 (1996).

[41] J. Braun, K. Miyamoto, A. Kimura, T. Okuda, M. Donath, H. Ebert, and J. Minár, New J. Phys. 16, 015005 (2014).

[42] J. Braun, in Band-Ferromagnetism: Ground-State and FiniteTemperature Phenomena, edited by K. Baberschke, M. Donath, and W. Nolting, Lecture Notes in Physics Vol. 580 (Springer, Berlin, 2001), p. 267.

[43] G. Malmstrøm and J. A. Rundgren, Comput. Phys. Commun. 19, 263 (1980).

[44] J. F. L. Hopkinson, J. B. Pendry, and D. J. Titterington, Comput. Phys. Commun. 19, 69 (1980).

[45] A. Nuber, J. Braun, F. Forster, J. Minar, F. Reinert, and H. Ebert, Phys. Rev. B 83, 165401 (2011).

[46] P. M. Echenique and J. B. Pendry, J. Phys. C 11, 2065 (1978).

[47] E. G. McRae, Rev. Mod. Phys. 51, 541 (1979). 